\title{
High Precision Current Ferrite Monitors
}

\author{
R.S. Shinde and M.G. Karmarkar \\ Accelerator Programme, MPS-1, Accelerator, Centre for Advanced Technology (CAT), Indore 452013, \\ India
}

\begin{abstract}
A high precision \& non-destructive types of current monitors using $\mathrm{Ni}-\mathrm{Zn}$ ferrite toroids for the measurements of electron beam currents has been developed. This monitoring system consists of $\mathrm{Ni}-\mathrm{Zn}$ ferrite toroidal cores, pickup coils, electromagnetic shields, a monitoring housing, current amplifiers \& ceramic ducts. The fast current monitors showed fast rise \& fall times ( $<3 \mathrm{~ns})$, the linearity within $2 \%$, the high sensitivity $(0.05 \mathrm{~V} / \mathrm{mA}$ at $50 \Omega$ load $)$ \& good $\mathrm{S} / \mathrm{N}$ ratio.
\end{abstract}

\section{INTRODUCTION}

Indus-l is a Synchrotron radiation source which consists of $20 \mathrm{MeV}$ microtron ( S-band), $700 \mathrm{MeV}$ Booster Synchrotron \& $450 \mathrm{MeV}$ Storage ring. Accelerator operation requires a real time device that (1) can measure low intensity beams, (2) is non intercepting, (3) has high gain stability (4) is independent of the beam energy \& $(5)$ can measure fast $e^{-}$beam currents $(<3 \mathrm{~ns})$. Non intercepting current monitors are used to tune \& operate the accelerator as well as to measure the beam currents delivered to the various stations $[1,2]$.

The characteristics required for the core monitors are a) fast rise \& fall times, b) high sensitivity, c) good linearity, d) good S/N ratio, e) wide frequency response (dc-250 MHz ).

This paper presents types of current monitors, their principles, constructional details \& their performance during injection \& acceleration.

\section{BEAM CURRENT MONITORS}

The particle beam passes through the centre hole of a toroidal core made of high permeability Ni-Zn ferrite. The beam can therefore be considered as a single tum primary winding of this toroidal transformer. The core carries a secondary winding which is terminated in the remote load resistance $R$. The magnetic field component, concentric to the beam, couples via the toroidal core \& induces a signal in the secondary winding. The current delivered by the secondary is given by:

$$
I_{S}=I_{B} / N, I_{B}=\text { Beam intensity }
$$

The low value of secondary turns $(N)$, permeability $(\sim 200)$ \& high frequency response $(>200$ $\mathrm{MHz}$ ) of ferrite core is choosen to keep minimum stray capacitance \& leakage inductance for fast rise time beam bunches measurements. Fig. 1 shows the structure of the high sensitivity current monitor which is mounted on the outside of a ceramic beam duct in the atmosphere. There are three fast rise time current monitors ( $<3 n s) \&$ two wall current monitors $(\sim 0.5$ ns rise time) are installed in the transfer line $-1,2$ \& Booster ring.

\section{0 MONITOR CALIBRATION}

Co-axial stand was built for development \& calibration of current monitors. The sensitivity of the ferrite monitors was tested using a tapered co-axial test stand \& tektronix probe. The positional response was measured by means of current pulse passing through wire in both horizontal \& vertical directions. 


\section{RESULTS}

The monitor system was tested using the $20 \mathrm{MeV}$ microtron \& $500 \mathrm{MeV}$ Booster synchrotron. The measurements were made using 4-channel digitizing oscilloscope (Le-Croy, $500 \mathrm{MHz}$ sampling). Semirigid cables are used for monitoring beam pulses into control room. Fig.2 shows the typical oscilloscope traces of 10 ns pulses detected by the monitor. The rise \& fall times are about 3 ns.

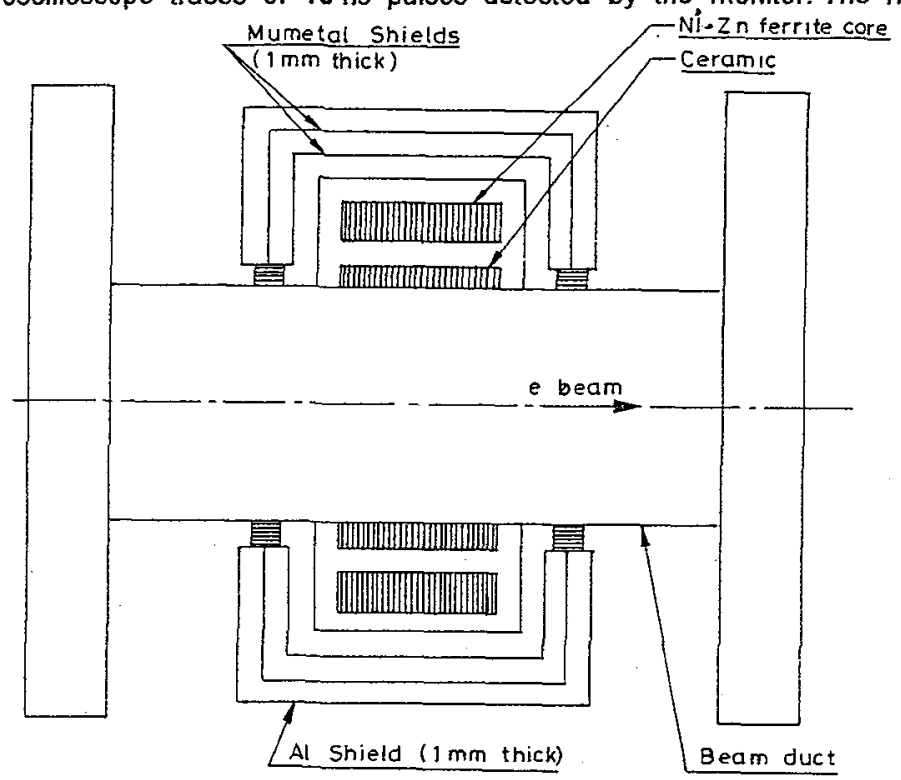

Fig.1. Schematic Cross section of beam current ferrite monitor

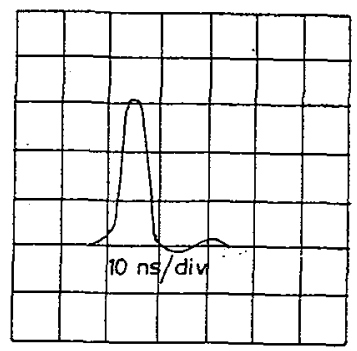

Test current pulse

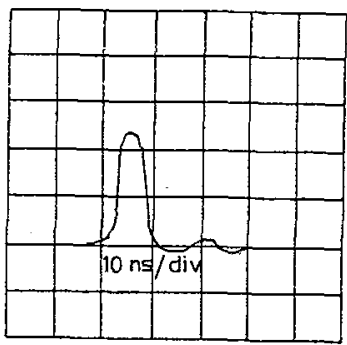

Ferrite core monitor

Fig.2. Typical oscilloscope traces of $10 \mathrm{~ns}$ pulses

\section{CONCLUSION}

The beam intensity of a pulse ( $10 \mathrm{~ns} \sim 1 \mu \mathrm{sec}$ ) beam is monitored by fast current ferrite monitors ( $<3 \mathrm{~ns})$. The beam pulse width \& peak current used were about 10 ns \& $100 \mathrm{~mA}$, respectively. The monitors showed the fast rise $\&$ fall times $(<3 \mathrm{~ns})$, the high sensitivity $(0.05 \mathrm{~V} / \mathrm{mA}, 50$ load), the linearity within $2 \%$ and good $S / N$ ratio due to the high pulse permeability of $\mathrm{Ni}$-Zn ferrite cores. A wall current monitors $(0.5$ ns ) are developed for a short pulse beam ( $\sim 2$ ns $)$ \& now under calibrations. The present current ferrite monitors is latest in sequence of evolving system based on high frequency ferrite toroids. The system provides fast beam currents. This is used as a diagnostic tool in tuning of accelerator. The system requires no operating adjustments and has proved stability, accuracy and reliability during a year of operation.

\section{Acknowledgements}

We would like to thank Dr. D.D. Bhawalkar, Director, CAT for his encouragements throughout this developmental works and my collegues Yadav \& Shiny for their technical assistance.

\section{References}

1. CAT Internal report, Dec. 95, "Development of magnetic devices for Accelerators"

2. C.Jenson \& J. Dinkel, IEEE Transactions on Magnetics, vol-31, No.1 (1995), 804-809 " Comparision of ferrite Materials for Pulse applications 